\title{
Average weighted trapping time of the node- and edge- weighted fractal networks
}

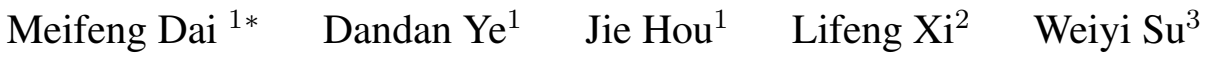 \\ ${ }^{1}$ Nonlinear Scientific Research Center, Faculty of Science, Jiangsu University \\ Zhenjiang, Jiangsu, 212013, P.R. China \\ ${ }^{2}$ Institute of Mathematics, Zhejiang Wanli University, Ningbo 315100, P.R. China \\ ${ }^{3}$ Department of Mathematics, Nanjiang University, Nanjing, 210093, P. R. China
}

\begin{abstract}
In this paper, we study the trapping problem in the node- and edge- weighted fractal networks with the underlying geometries, focusing on a particular case with a perfect trap located at the central node. We derive the exact analytic formulas of the average weighted trapping time (AWTT), the average of node-to-trap mean weighted first-passage time over the whole networks, in terms of the network size $N_{g}$, the number of copies $s$, the node-weight factor $w$ and the edge-weight factor $r$. The obtained result displays that in the large network, the AWTT grows as a power-law function of the network size $N_{g}$ with the exponent, represented by $\theta(s, r, w)=\log _{s}\left(s r w^{2}\right)$ when $s r w^{2} \neq 1$. Especially when $s r w^{2}=1$, AWTT grows with increasing order $N_{g}$ as $\log N_{g}$. This also means that the efficiency of the trapping process depend on three main parameters: the number of copies $s>1$, node-weight factor $0<w \leq 1$, and edge-weight factor $0<r \leq 1$. The smaller the value of $s r w^{2}$ is, the more efficient the trapping process is.
\end{abstract}

Keywords: Fractal network; The node- and edge-weighted network; Average weighted trapping time; Weight factor.

\section{Introduction}

In the last few years, complex networks have attracted a growing interest from a wide circle of researchers [1]. Weighted networks are the extension of unweighted networks. Node weight represents the importance of a node while edge weight represents the the intensity of a link. Node weights and edge weights are ubiquitous for graph representations of complex systems[2]. For example, node weights can represent volumes of the regions in brain networks [3,4], surface areas in climate networks [5, 6], market values in world trade networks [7, 8], sizes of the IP address space in the world-wide-web networks and article sizes in Wikipedia $[9,10,11]$, the different dynamical behaviors in engineering and biological networks $[12,13]$ and the amount of external input signal in the chaotic system $[14,15]$. Practical realizations of edge weights in real networks range from the number of passengers traveling yearly between two airports in airport networks [16], the connection between two cells in cellular neural network[17], to the intensity of predator-prey interactions in ecosystems [18] or the traffic measured in packets per unit time between routers in the Internet [19].

On the basis of the weighted fractal networks [20], the node weights and the edge weights, we will propose the node- and edge- weighted fractal networks. The node- and edge- weighted fractal networks have been generalized for applications in the domain of many realistic networks, i.e., the highway network. In the highway networks, nodes represent the highway passenger station and links indicate that there are non-stop vehicle between any two highway passenger stations. In the node- and edge- weighted fractal networks, we study that trapping is a paradigmatic one, which is a kind of node-weight-dependent walk with a deep trap fixed at a given position, absorbing all walkers that visit it. A basic quantity relevant to the trapping problem is the weighted trapping time (WTT), commonly called the mean weighted firstpassage time (MWFPT), for the node-weight-dependent walk. For two adjacency nodes $i$ and $j$, we define the weighted time is the corresponding edge weight $r_{i j}$ [21]. The WTT for a node $i$, denoted by $F_{i}$, is the weighted time for a walker starting from node $i$ to reach the trap for the first time. The average weighted trapping time (AWTT), $\langle T\rangle_{g}$, is defined as the average of $F_{i}$ over all source nodes in the system other than the trap, which provides a useful indicator for the efficiency of trapping.

E-mail address: daimf@mail.ujs.edu.cn 
In this paper, we introduce a class of node- and edge- weighted fractal networks depending on three main parameters: the number of copies $s>1$, node-weight factor $0<w \leq 1$, and edge-weight factor $0<r \leq 1$. We study analytically the node-weight-dependent walk on the node- and edge- weighted fractal networks, which is a fundamental process gaining considerable recent attention. And we define the average weighted trapping time (AWTT) on node-edge weighted networks. So we derive exactly the AWTT formula, which displays that in large networks, the leading behaviors of AWTT for the node- and edge- weighted fractal networks follow distinct scalings, with the trapping efficiency associated with the network size $N_{g}$, the number of copies $s$, the node-weight factor $w$ and the edge-weight factor $r$.

\section{The node- and edge- weighted fractal networks}

It is well known that one of the most amazing and interesting feature of fractals is their self-similarity, namely looking at all scales we can find conformal copies of the whole set. Starting from this property one can provide rules to build up fractals as fixed points of iterated function systems, IFS for short[22, 23]. So iteration methods are one way to achieve the self-similarity exhibited by fractals $[24,25]$. Intuited by weighted fractal networks and the highway networks, the node- and edge- weighted fractal networks are constructed in an iterative method.

We choose one typical node weight which is the passenger-capacity weight of the highway passenger station and one typical edge weight which is the consuming traveling cost. Denote by $w_{i j}$ the node weight connecting the nodes $i$ and $j$, and denote by $r_{i j}$ the edge weight connecting the nodes $i$ and $j$. Let $w_{i j}$ be related to the weight factor $w$, and let $r_{i j}$ be related to the weight factor $r$. The main goal of this section is aimed at constructing the node- and edge- weighted fractal networks depending on the three main parameters: the number of copies $s$, node-weight factor $w$, and edge-weight factor $r$. Moreover taking advantage of the similarity with the IFS fractals, the node- and edge- weighted fractal networks are defined as follows.

Let us fix two positive real numbers $0<w \leq 1,0<r \leq 1$ and a positive integer $s>1$. Denote by $w$ the node-weight factor and denote by $r$ the edge-weight factor. Starting with a given initial network $G$, we can construct a family of node- and edge- weighted fractal networks. Let $G_{g}(w, r)(g \geq 1)$ denote the nodeand edge- weighted fractal networks after $g$ iterations (generations). The node set $A_{g}$ of $G_{g}(w, r)$ will be classed into $g+1$ node-sets, $A_{g}=A_{g, 0} \cup A_{g, 1} \cup \cdots \cup A_{g, g}=\bigcup_{i=0}^{g} A_{g, i}(g=0,1,2, \cdots)$.

(i) At the initial generation $(g=0), G_{0}(w, r)$ is a given initial network $G$ containing $d+1$ nodes. One node of $G$ has been labeled attaching node and denoted by $a$ while all other are labeled consecutively as $a_{1}, a_{2}, \cdots, a_{d}$, where Node $a$ has the $d$ neighboring nodes, $a_{1}, a_{2}, \cdots, a_{d}$, satisfying the symmetry of nodes in $G$. Each node has unitary node-weight and each edge has unitary edge weight in $G$. Let the node-set $A_{0,0}=\left\{a, a_{1}, a_{2}, \cdots, a_{d}\right\}$.

Remark: The symmetry of nodes $a_{1}, a_{2}, \cdots, a_{d}$ in $G$ means that the network $G$ is invariable no matter how two arbitrary nodes $a_{i}$ and $a_{j}$ are exchanged.

(ii) At $g=1, G_{1}(w ; r)$ has one central node labelled by $a_{1,0}$ with unitary node weight. Let $G_{0}^{(1)}(w, r)$, $G_{0}^{(2)}(w, r), \cdots, G_{0}^{(s)}(w, r)$ be $s$ copies of $G_{0}(w, r)$, whose weighted nodes and weighted edges have been scaled by a node-weight factor $w$ and a edge-weight factor $r$, respectively. $A_{0,0}^{(i)}(i=1,2, \cdots, s)$ is the copy set of $A_{0,0}$. For $i=1,2, \cdots, s$, let us denote by $a^{(i)}$ the node in $G_{0}^{(i)}(w, r)$ image of the labeled node $a \in G_{0}(w, r)$, then link all those labeled nodes $a^{(i)}(i=1,2, \cdots, s)$ to the central node $a_{1,0}$ through edges of unitary weight, then the connected network $G_{1}(w, r)$ is obtained. Let $A_{1}=A_{1,0} \cup A_{1,1}$, where $A_{1,0}=\left\{a_{1,0}\right\}, A_{1,1}=\bigcup_{i=1}^{s} A_{0,0}^{(i)}$.

(iii) For any $g \geq 1, G_{g+1}(w, r)$ is obtained from $G_{g}(w, r)$ (see Fig. 1): $G_{g+1}(w, r)$ has one central node labelled by $a_{g+1,0}$ with unitary node weight. Let $G_{g}^{(1)}(w, r), G_{g}^{(2)}(w, r), \cdots, G_{g}^{(s)}(w, r)$ be $s$ copies of $G_{g}(w, r)$, whose weighted nodes and weighted edges have been scaled by a node-weight factor $w$ and a edge-weight factor $r$, respectively. $A_{g, j}^{(i)}(i=1,2, \cdots, s)$ is the copy set of $A_{g, j}(j=0,1, \cdots, g)$. For $i=1,2, \cdots, s$, let us denote by $a_{g, 0}^{(i)}$ the node in $G_{g}^{(i)}(w, r)(i=1,2, \cdots, s)$ image of the labeled node 
$a_{g, 0} \in G_{g}(w, r)$, then link all those labeled nodes $a_{g, 0}^{(i)}(i=1,2, \cdots, s)$ to the central node $a_{g+1,0}$ through edges of unitary weight, then the connected network $G_{g+1}(w, r)$ is obtained. Let $A_{g+1}=$ $A_{g+1,0} \cup A_{g+1,1} \cup \cdots \cup A_{g+1, g+1}$, where $A_{g+1,0}=\left\{a_{g+1,0}\right\}, A_{g+1,1}=\bigcup_{i=1}^{s} A_{g, 0}^{(i)}, \cdots, A_{g+1, g}=$ $\bigcup_{i=1}^{s} A_{g, g-1}^{(i)}, A_{g+1, g+1}=\bigcup_{i=1}^{s} A_{g, g}^{(i)}$.

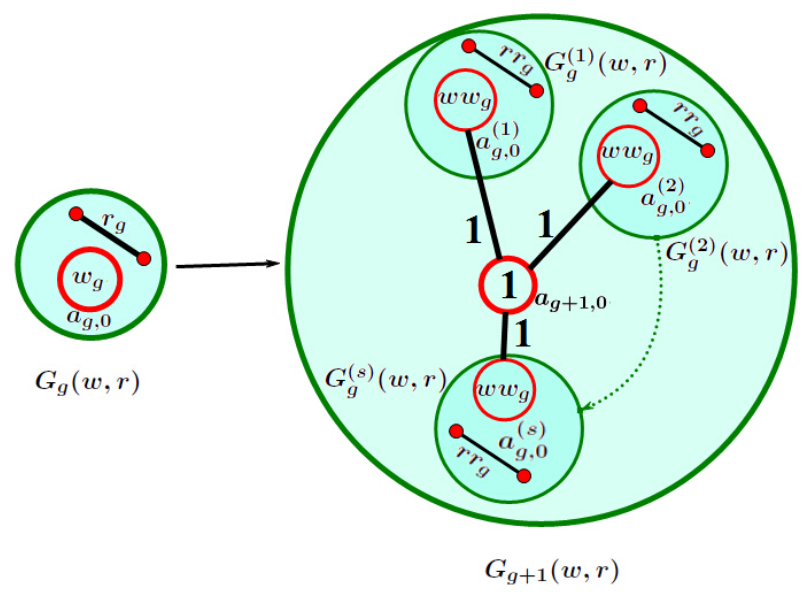

Figure 1: Construction method of the node- and edge- weighted fractal networks from $g$ to $g+1$.

The general node-and edge- weighted fractal networks $G_{g+1}(w, r)$ are defined by the iterative construction method. In order to completely explain the general node-and edge- weighted networks, we present two special node-and edge- weighted fractal networks: the 'Sierpinski' node- and edge- weighted fractal networks when $s=3$ (see Fig. 2) and the 'Cantor dust' node- and edge- weighted fractal networks when $s=4$ (see Fig. 3).

By the construction, we can easily obtain some basic quantities, which are very useful for computing some quantities which we are concerned with in this paper. Let $N_{g}=\left|A_{g}\right|=\left|A_{g, 0}\right|+\left|A_{g, 1}\right|+\cdots+\left|A_{g, g}\right|$ be the total number of nodes in $G_{g}(w, r)$. Then, it is easy to verify that $\left|A_{g, 0}\right|=1,\left|A_{g, 1}\right|=s, \cdots,\left|A_{g, g-1}\right|=$ $s^{g-1},\left|A_{g, g}\right|=s^{g}(d+1)$. Thus, the total number of nodes in $G_{g}(w, r)$ is

$$
N_{g}=\sum_{i=0}^{g}\left|A_{g, i}\right|=1+s+\cdots+s^{g-1}+s^{g}(d+1)=s^{g}(d+1)+\frac{s^{g}-1}{s-1} \approx \frac{d s+s-d}{s-1} s^{g} .
$$

\section{Structural features of node- and edge- weighted fractal networks}

Carletti et al. [20] have defined a class of edge-weighted complex networks sharing several properties with fractal sets, which depend on two main parameters: the number of copies $s>1$ and edge-weight factor $0<r \leq 1$. Its topology can be completely analytically characterized in terms of the involved parameters and of the fractal dimension, $d_{\text {fract }}=-\frac{\log s}{\log r}$. Wei et al. [26] proposed an improved boxcovering algorithm for weighted networks (BCANw). The fractal dimension obtained by using BCANw is closer to the similarity dimension of the 'Sierpinski' edge-weighted fractal networks in Ref. 15. Carletti et al. [20] characterized the topology of edge-weighted fractal networks $G_{g}(1, r)$, by analytically studying their properties such as the average degree, the node strength distribution, the average weighted shortest path and the average weighted clustering coefficient. It has been shown that the node strength distribution and average weighted clustering coefficient depend on the fractal dimension of the IFS fractal; for instance the networks are scale-free, the exponent being the fractal dimension. These edge-weighted fractal networks 

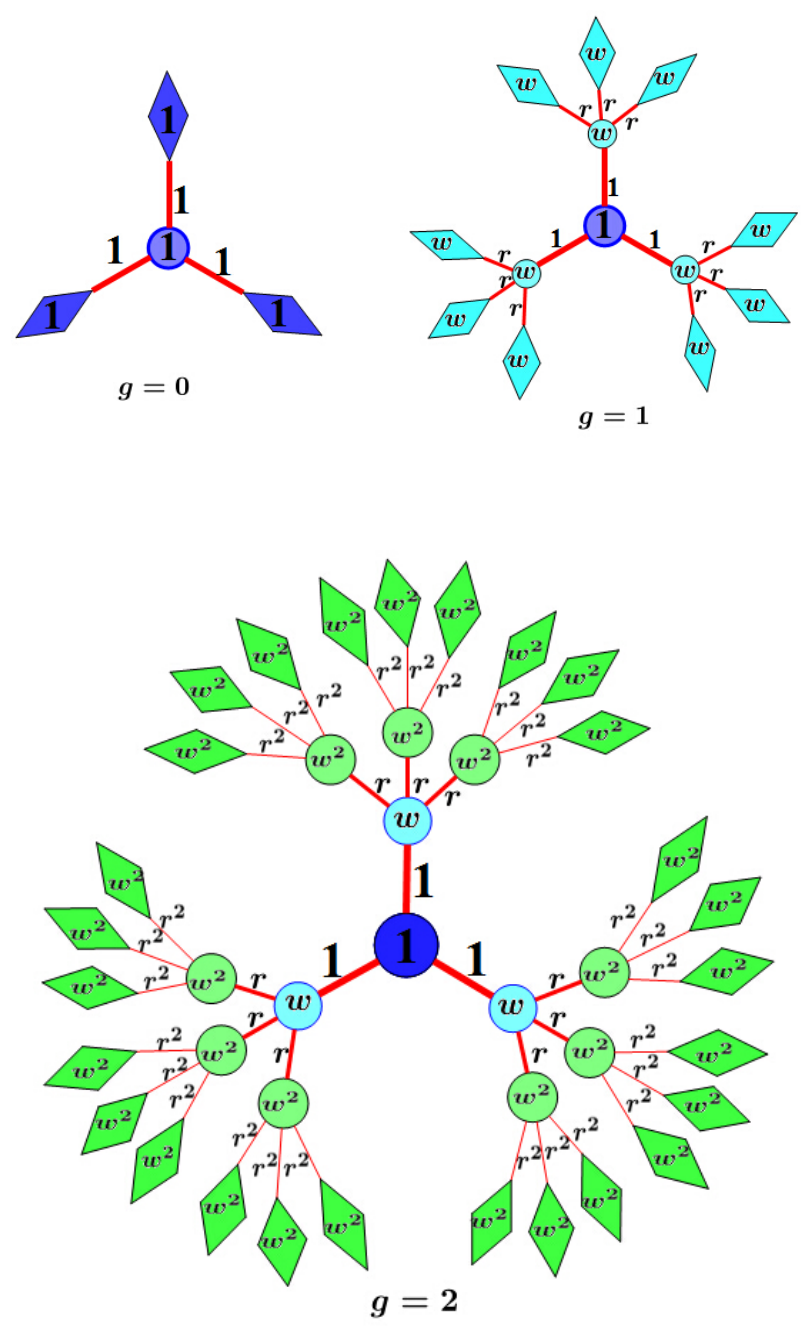

Figure 2: Iterative construction method for the 'Sierpinski' node- and edge- weighted fractal networks at iterations $g=0,1,2$. A circles or a diamond represents a node. Take $g=2$, for example, the royal blue circle belongs to $A_{2,0}$, blue-green circles belong to $A_{2,1}$, green circles or diamonds belong to $A_{2,2}$.

exhibit the small-world property because the average shortest path increases logarithmically with the system size and the clustering coefficient is asymptotically constant.

Based on the edge-weighted fractal networks, we will further study the node- and edge- weighted fractal networks. In the node- and edge- weighted fractal networks, node weights do not affect their topology properties such as the average degree, the node strength distribution, the average weighted shortest path and the average weighted clustering coefficient. It is well known that the algorithms for fractal dimension of complex networks is various, particularly the box covering algorithm [27, 28, 29]. Due to box-covering algorithm for fractal dimension of complex networks, node weights do not affect the fractal dimension. So fractal dimension of the node- and edge- weighted fractal networks is still equivalent to $d_{\text {fract }}=-\frac{\log s}{\log r}$.

\section{Trapping with a single trap fixed at the central node}

Diffusion is a key element of a large set of phenomena occurring on natural and social systems modeled in terms of weighted complex networks. Assuming that the diffusion process is local, there are three most 


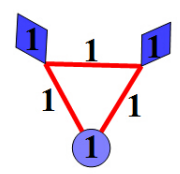

$g=0$
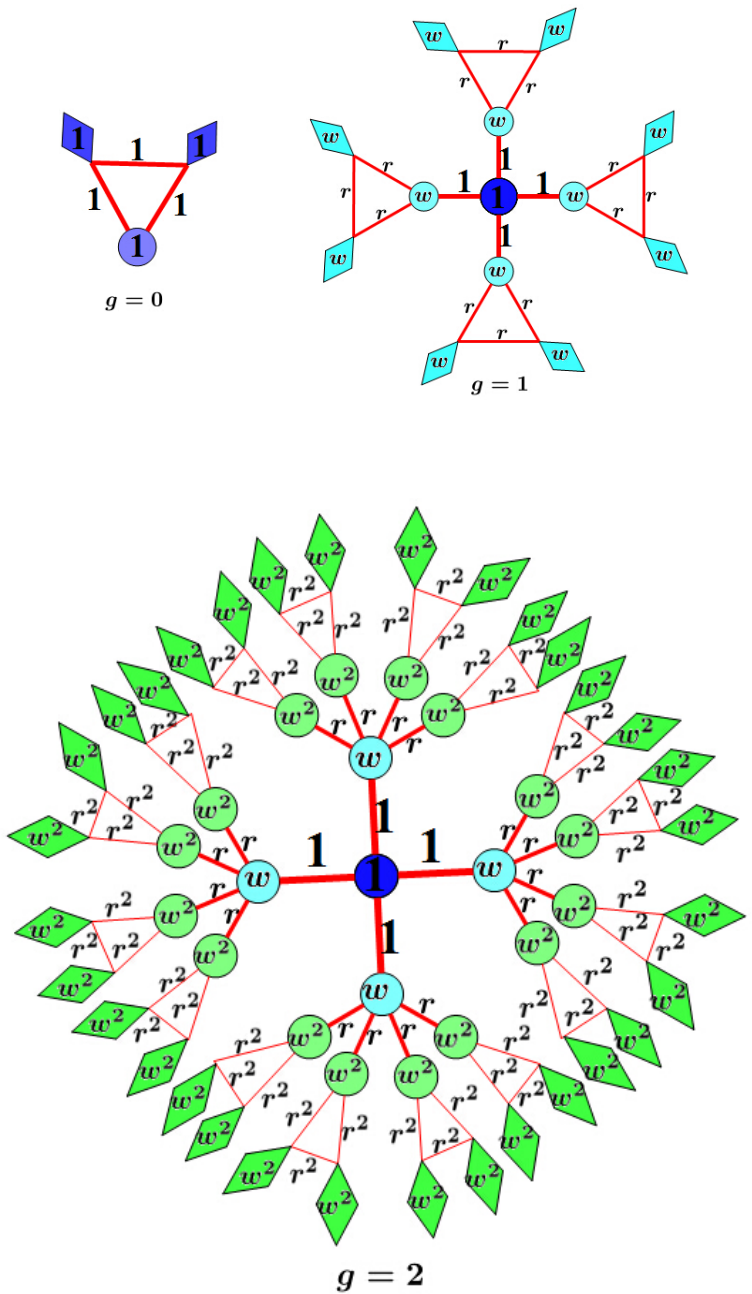

Figure 3: Iterative construction method for the 'Cantor dust' node- and edge- weighted fractal networks at iterations $g=0,1,2$. A circles or a diamond represents a node. Take $g=2$, for example, the royal blue circle belongs to $A_{2,0}$, blue-green circles belong to $A_{2,1}$, green circles or diamonds belong to $A_{2,2}$.

general kinds of random walks: random walk, weigh-dependent walk and strength-dependent walk. A random walker may choose one of its neighboring edges at the same probability i.e., random walk [30] . In edge-weighted networks, however, the walker may choose an edge according to its weight or the strength of the node connected by it, i.e., weight-dependent walk or strength-dependent walk.

To take different sizes of nodes into account, it is useful to assign an individual weight to every node. Taking the highway network, for example, nodes represent the highway passenger station and links indicate that there are non-stop vehicle between any two highway passenger stations. Passenger throughput of the highway passenger station is firstly taken into account when a passenger chooses one highway passenger station to the other. So node weight is the passenger throughput of the highway passenger station. A passenger arrives at the next destination station with probability proportional to the passenger throughput of the highway passenger station. To take practical realizations of node weights in real networks, a walker will choose an edge according to the node weight of its nearest neighbors, named node-weight-dependent walk. The node-weight-dependent walk here is an novel way. For node-weight-dependent walk, a walker chooses one of its nearest neighbors with probability proportional to the node weight of neighboring nodes.

After introducing the node- and edge- weighted fractal networks, in this section we study the trapping 
problem performed on $G_{g}(w, r)$ with the node-weight-dependent walk, where a single immobile trap is located at Node $a_{g, 0}$. At each discrete time step, the walker jumps from its current Node $m \in A_{g, i}$ to any of its neighboring Node $n \in A_{g, j}(|i-j|=1, i, j=0,1,2, \cdots, g-1)$ with the probability $p_{m \rightarrow n}=\frac{w_{n}}{s_{m}}$, where $s_{m}$ is the sum of the weights of the neighboring nodes of the Node $m$, i.e., $s_{m}=\sum_{n \in \nu(m)} w_{n}$, where the index $n$ runs over the set $\nu(m)$ of neighbors of Node $m$.

The transition probability from Node $m \in A_{g, i}$ to its neighbor $n \in A_{g, j}$ is

$$
p_{m \rightarrow n}=\frac{w_{n}}{w^{i-1}+3 w^{i+1}}= \begin{cases}\frac{w^{i-1}}{w^{i-1}+s w^{i+1}}=\frac{1}{1+s w^{2}}, & j=i-1 \\ \frac{s w^{i+1}}{w^{i-1}+s w^{i+1}}=\frac{s w^{2}}{1+s w^{2}}, & j=i+1 .\end{cases}
$$

Still taking the highway network, for example, a link is created if a passenger may want to directly reach the destination station from the starting station. A passenger can choose different routes from his origin to the destination according to the consuming traveling cost. So edge weight is the total value of the consuming traveling cost in the highway network. For two adjacency nodes $i$ and $j$, we define the weighted time is the corresponding edge weight $r_{i j}$. Let $F_{i}(g)$ denote the weighted trapping time (WTT) for node $i$, which is the mean weighted first-passage time (MWFPT) for a walker staring from node $i$ of $A_{g, i}(i=0,1, \cdots, g)$ to first arrive at the trap in $G_{g}(w, r)$. The most important quantity related to the trapping problem is the AWTT, $\langle T\rangle_{g}$, which is the average of $F_{i}(g)$ over all starting nodes distributed uniformly over the whole network. By definition, $\langle T\rangle_{g}$ is given in $G_{g}(w, r)$ by

$$
\langle T\rangle_{g}=\frac{1}{N_{g}-1} \sum_{j=1}^{g} \sum_{i \in A_{g, j}} F_{i}(g) .
$$

In the sequel, we will determine explicitly this $\langle T\rangle_{g}$ for $G_{g}(w, r)$, and show how $\langle T\rangle_{g}$ scales with the system size, node-weight factor $w$ and edge-weight factor $r$ so as to get information on the internal structure of the node- and edge- weighted fractal networks and explore the effect of the underlying geometry, node weights and edge weights on the trapping efficiency of the node- and edge- weighted fractal networks.

In the given initial network $G$, let $F_{a_{i}}$ be the the mean weighted first-passage times (MWFPTs) for a walker from Node $a_{i}$ to the attaching node $a$. Here, we denote by $T_{t o t}(0)$ the sum of MWFPTs for all nodes to the attaching node $a$, i.e., $T_{t o t}(0)=\sum_{i=1}^{d} F_{a_{i}}$. Because of the symmetry of nodes $a_{1}, a_{2}, \cdots, a_{d}$, $F_{a_{1}}=F_{a_{2}}=\cdots=F_{a_{d}}$ and $F_{a_{i}}=\frac{T_{t o t}(0)}{d} . T_{t o t}(0)$ is a constant number for the given initial network $G$. Let $G\left(w^{g}, r^{g}\right)$ be the copy of the initial networks $G$, whose weighted nodes and weighted edges have been scaled by a node-weight factor $w^{g}$ and a edge-weight factor $r^{g}$, respectively (see Figs.4 and 5). In $G\left(w^{g}, r^{g}\right)$, the corresponding $d+1$ nodes are labelled consecutively as $b, b_{1}, b_{2}, \cdots, b_{d}$. Let $F_{b_{i}}$ be the the mean weighted first-passage times (MWFPTs) for a walker from Node $b_{i}$ to the attaching node $b$. It is obvious that $\sum_{i=1}^{d} F_{b_{i}}=r^{g} \sum_{i=1}^{d} F_{a_{i}}=r^{g} T_{t o t}(0)$.

The quantities below are discussed in $G_{g}(w, r)$. All nodes belonging to the $i$-th set $A_{g, i}(i=0,1, \cdots, g-$ 1) have the same WTT. $F_{i}(g)(i=0,1, \cdots, g-1)$ represents the WTT starting from a node in the $i$-th set in $G_{g}(w, r)$. The node set $A_{g, g}$ is the union of $s^{g}$ node sets, one of which belongs to the network $G\left(w^{g}, r^{g}\right)$. Let $F_{g}(g)$ be used to represent the WTT starting from an attaching node $b$ in $G\left(w^{g}, r^{g}\right)$ to the central node $a_{g, 0}$ and $F_{g}^{\prime}(g)$ is used to represent the WTT starting from any one node $b_{i}$ in $G\left(w^{g}, r^{g}\right)$ to the central node $a_{g, 0} . F_{i}(g)(i=0,1, \cdots, g)$ satisfies the following relations:

$$
F_{i}(g)=\left\{\begin{array}{cl}
0 & , i=0 \\
\frac{1}{1+s w^{2}}\left[r^{i-1}+F_{i-1}(g)\right]+\frac{s w^{2}}{1+s w^{2}}\left[r^{i}+F_{i+1}(g)\right] & , 0<i<g \\
\frac{1}{1+d w}\left[r^{g-1}+F_{g-1}(g)\right]+\frac{d w}{1+d w}\left[r^{g}+F_{g}^{\prime}(g)\right] & , i=g .
\end{array}\right.
$$

Eq. (4) can be elaborated as follows:

(i) Eq. (4) is obvious when $i=0$. 

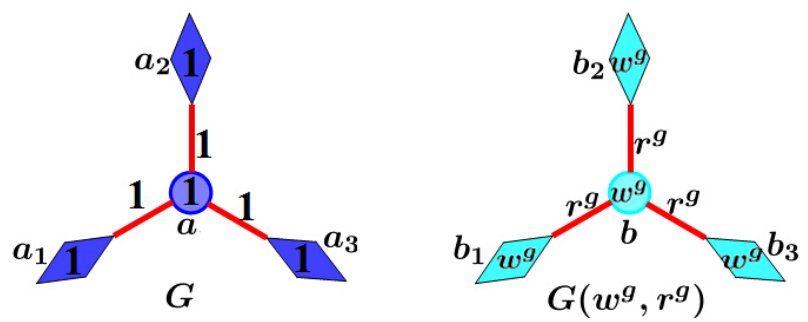

Figure 4: A circle represents an attaching node and the diamonds represents some other nodes rather than attaching nodes in $G$ and in $G\left(w^{g}, r^{g}\right)$ in the 'Sierpinski' node- and edge- weighted fractal networks .
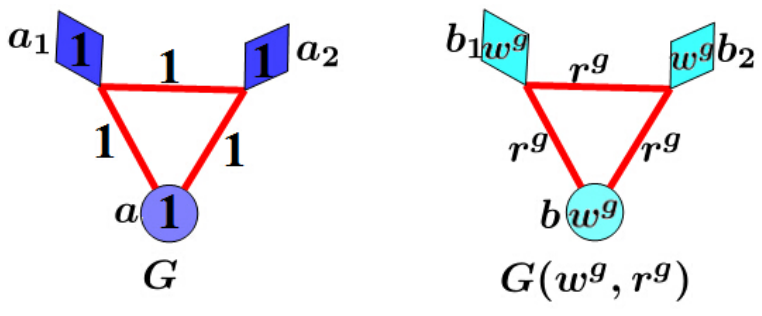

Figure 5: A circle represents an attaching node and the diamonds represents some other nodes rather than attaching nodes in $G$ and in $G\left(w^{g}, r^{g}\right)$ in the 'Cantor dust' node- and edge- weighted fractal networks.

(ii) When $0<i<g$, the first term on the right-hand side accounts for the case that with probability $\frac{1}{1+s w^{2}}$ (see Eq. (2)) the walker starting from a node in the $i$-th set $A_{g, i}$ first takes $r^{i-1}$ weighted time to arrive at its unique neighbor in the $i-1$-th set $A_{g, i-1}$ and then takes $F_{i-1}(g)$ steps to reach the trap for the first time. The second term explains the fact that with probability $\frac{s w^{2}}{1+s w^{2}}$ (see Eq. (2)) the walker first takes $r^{i}$ weighted time to a node in the $i+1-t h$ set $A_{g, i+1}$ and then jumps $F_{i+1}(g)$ more steps to first reach the central node.

(iii) When $i=g$, the first term accounts for the case that with probability $\frac{w^{g-1}}{w^{g-1}+d w^{g}}=\frac{1}{1+d w}$ the walker starting from one node of $s^{g}$ attaching nodes of the node set $A_{g, g}, b$, first takes $r^{g-1}$ weighted time to arrive at its unique neighbor in the $g-1$-th set $A_{g, g-1}$ and then takes $F_{g-1}(g)$ steps to reach the trap for the first time; the second term explains the fact that with probability $\frac{d w^{g}}{w^{g-1}+d w^{g}}=\frac{d w}{1+d w}$ the walker starting from the node $b$ first takes $r^{g}$ weighted time to certain node $b_{i}$ in one $G\left(w^{g}, r^{g}\right)$ and then jumps $F_{g}^{\prime}(g)$ more steps to first reach the central node.

From Eq. (4), we can obtain the following relation:

$$
F_{i}(g)-F_{i-1}(g)=\left(1+s r w^{2}\right) r^{i-1}+s w^{2}\left[F_{i+1}(g)-F_{i}(g)\right], i=1,2, \cdots, g-1 .
$$

Let $A_{j}(g)=F_{g-j}(g)-F_{g-j-1}(g)(j=g-i)$. Then,

$$
A_{j}(g)=\left(1+s r w^{2}\right) r^{g-j-1}+s w^{2} A_{j-1}(g)
$$

holds for all $0<j<g$. 
For $i=g, A_{0}(g)$ can be deduced from Eq. (4), that is $A_{0}(g)=F_{g}(g)-F_{g-1}(g)=(1+d r w) r^{g-1}+$ $d w\left(F_{g}^{\prime}(g)-F_{g}(g)\right)=(1+d r w) r^{g-1}+d w F_{b_{i}}=(1+d r w) r^{g-1}+d w r^{g} F_{a_{i}}=\left(1+d r w+d r w \frac{T_{t o t}(0)}{d}\right) r^{g-1}$.

(1) If $s r w^{2}=1$, Eq. (5) can be solved to yield

$$
A_{j}(g)=2 j r^{g-j-1}+\left(1+d r w+d r w \frac{T_{t o t}(0)}{d}\right) r^{g-j-1} .
$$

Then for $j<g$, we can obtain

$$
F_{g-j}(g)=F_{g-j-1}(g)+2 j r^{g-j-1}+\left(1+d r w+d r w \frac{T_{t o t}(0)}{d}\right) r^{g-j-1},
$$

which leads to

$$
F_{i}(g)=F_{i-1}(g)+2(g-i) r^{i-1}+\left(1+d r w+d r w \frac{T_{t o t}(0)}{d}\right) r^{i-1},
$$

for all $0<i \leq g$. Using the initial condition $F_{0}(g)=0$, Eq. (6) can be solved to yield

$$
F_{i}(g)=2 g \frac{1-r^{i}}{1-r}+c_{1} \frac{1-r^{i}}{1-r}+\frac{2}{1-r} i r^{i}, \quad i=1,2, \cdots, g,
$$

where $c_{1}=d r w\left(1+\frac{T_{t o t}(0)}{d}\right)-\frac{1+r}{1-r}$.

Then, according to Eq. (3), the explicit expression for ATT for the trapping problem in $G_{g}(w, r)$ can be obtained as

$$
\begin{aligned}
\langle T\rangle_{g}= & \frac{\sum_{i=1}^{g-1}\left[\left|A_{g, i}\right| \times F_{i}(g)\right]+\left|A_{g, g}\right| \times F_{i}(g)+s^{g} \times r^{g} T_{t o t}(0)}{N_{g}-1} \\
= & {\left[\frac{2}{(1-r)(s-1)}+\frac{2(d+1)}{1-r}\right] \frac{g s^{g}}{N_{g}-1}-\left[\frac{c_{1}}{(1-r)(s r-1)}+\frac{c_{1}(d+1)}{1-r}+\frac{2 s r}{(1-r)(s r-1)^{2}}\right.} \\
& \left.+T_{t o t}(0)\right] \frac{(s r)^{g}}{N_{g}-1}+\left[\frac{c_{1}}{(1-r)(s-1)}+\frac{c_{1}(d+1)}{1-r}\right] \frac{s^{g}}{N_{g}-1}+\left[\frac{2 s r}{(1-r)(s r-1)}\right. \\
& \left.-\frac{2 s}{(1-r)(s-1)}\right] \frac{g}{N_{g}-1}+\left[\frac{c_{1} s r}{(1-r)(s r-1)}+\frac{2 s r}{(1-r)(s r-1)^{2}}-\frac{c_{1} s}{(1-r)(s-1)}\right] \frac{1}{N_{g}-1} \\
\approx & {\left[\frac{2}{(1-r)(s-1)}+\frac{2(d+1)}{1-r}\right] \frac{g s^{g}}{N_{g}-1}-\left[\frac{c_{1}}{(1-r)(s r-1)}+\frac{c_{1}(d+1)}{1-r}+\frac{2 s r}{(1-r)(s r-1)^{2}}\right.} \\
& \left.+T_{t o t}(0)\right] \frac{(s r)^{g}}{N_{g}-1},
\end{aligned}
$$

where $0<r<1, s r \neq 1$. We proceed to represent $\langle T\rangle_{g}$ as a function of the network size $N_{g}$. From Eq. (1), we have

$$
N_{g}-1 \approx \frac{d s+s-d}{s-1} s^{g}
$$

which enables to write $\langle T\rangle_{g}$ in the following form:

$$
\begin{aligned}
\langle T\rangle_{g} \approx & \frac{s-1}{d s+s-d}\left[\frac{2}{(1-r)(s-1)}+\frac{2(d+1)}{1-r}\right] g-\frac{s-1}{d s+s-d}\left[\frac{c_{1}}{(1-r)(s r-1)}+\frac{c_{1}(d+1)}{1-r}\right. \\
& \left.+\frac{2 s r}{(1-r)(s r-1)^{2}}+T_{t o t}(0)\right] r^{g} \\
\approx & \frac{s-1}{d s+s-d}\left[\frac{2}{(1-r)(s-1)}+\frac{2(d+1)}{1-r}\right] g \sim \log N_{g} .
\end{aligned}
$$


(2) If $s r w^{2} \neq 1$, Eq. (5) can be solved to yield

$$
A_{j}(g)=\frac{1+s r w^{2}}{1-s r w^{2}} r^{g-j-1}+c_{2} r^{g-1}\left(s w^{2}\right)^{j},
$$

where $c_{2}=d r w\left(1+\frac{T_{t o t}(0)}{d}\right)-\frac{2 s w^{2} r}{1-s r w^{2}}$.

Then for $j<g$, we get

$$
F_{g-j}(g)=F_{g-j-1}(g)+\frac{1+s r w^{2}}{1-s r w^{2}} r^{g-j-1}+c_{2} r^{g-1}\left(s w^{2}\right)^{j},
$$

which leads to

$$
F_{i}(g)=F_{i-1}(g)+\frac{1+s r w^{2}}{1-s r w^{2}} r^{i-1}+c_{2} r^{g-1}\left(s w^{2}\right)^{g-i},
$$

for all $0<i \leq g$. Using the initial condition $F_{0}(g)=0$, Eq. (9) can be solved to yield

$$
F_{i}(g)=\frac{1+s r w^{2}}{\left(1-s r w^{2}\right)(1-r)}\left[1-r^{i}\right]+\frac{c_{2}\left(s r w^{2}\right)^{g}}{r\left(s w^{2}-1\right)}\left[1-\frac{1}{\left(s w^{2}\right)^{i}}\right]
$$

for all $1 \leq i \leq g$.

Case $(i): 0<w<1$ and $0<r<1$

According to Eq. (3), the explicit expression for ATT for the trapping problem in $G_{g}(w, r)$ can be obtained as

$$
\begin{aligned}
\langle T\rangle_{g}= & \frac{\sum_{i=1}^{g-1}\left[\left|A_{g, i}\right| \times F_{i}(g)\right]+\left|A_{g, g}\right| \times F_{i}(g)+s^{g} \times r^{g} T_{t o t}(0)}{N_{g}-1} \\
= & \frac{c_{2}(d s+s-d)\left(r w^{2}\right)^{g}}{r\left(s w^{2}-1\right)(s-1)} \frac{\left(s^{g}\right)^{2}}{N_{g}-1}+\left[\frac{\left(1+s r w^{2}\right) r^{g}}{\left(1-s r w^{2}\right)(1-r)(1-s r)}-\frac{1+s r w^{2}}{\left(1-s r w^{2}\right)(1-r)(1-s)}\right. \\
& +\frac{c_{2} s\left(r w^{2}\right)^{g}}{r\left(s w^{2}-1\right)(1-s)}-\frac{c_{2}\left(r w^{2}\right)^{g}}{r\left(s w^{2}-1\right)\left(w^{2}-1\right)}+\frac{c_{2} w^{2} r^{g}}{r\left(s w^{2}-1\right)\left(w^{2}-1\right)}+r^{g} T_{t o t}(0) \\
& \left.-\frac{c_{2}(d+1) r^{g}}{r\left(s w^{2}-1\right)}+\frac{(d+1)\left(1+s r w^{2}\right)\left(1-r^{g}\right)}{\left(1-s r w^{2}\right)(1-r)}\right] \frac{s^{g}}{N_{g}-1}+\frac{s\left(1+s r w^{2}\right)}{\left(1-s r w^{2}\right)(1-s)(1-s r)} \frac{1}{N_{g}-1} \\
\approx & \frac{c_{2}(d s+s-d)\left(r w^{2}\right)^{g}}{r\left(s w^{2}-1\right)(s-1)} \frac{\left(s^{g}\right)^{2}}{N_{g}-1} .
\end{aligned}
$$

We proceed to represent $\langle T\rangle_{g}$ as a function of the network size $N_{g}$. From Eq. (1), we have

$$
N_{g}-1 \approx \frac{d s+s-d}{s-1} s^{g}
$$

which enables to write $\langle T\rangle_{g}$ in the following form:

$$
\langle T\rangle_{g} \approx \frac{c_{2}}{r\left(s w^{2}-1\right)}\left(s r w^{2}\right)^{g} .
$$

Eq. (11) provides the exact dependence relation of ATT on the network order $g$ and there parameters $w, r, s$. For a large system, i.e., $N_{g} \rightarrow \infty$, we have the following expression for the dominating term of $\langle T\rangle_{g}:$

$$
\langle T\rangle_{g} \approx \frac{c_{2}}{r\left(s w^{2}-1\right)}\left(\frac{s-1}{d s+s-d}\right)^{\log _{s}\left(s r w^{2}\right)} N_{g}^{\log _{s}\left(s r w^{2}\right)} \sim N_{g}^{\log _{s}\left(s r w^{2}\right)} .
$$

Case (ii) : $r=1$ and $0<w<1$ 
$\langle T\rangle_{g}$ can be obtained as

$$
\begin{aligned}
\langle T\rangle_{g}= & \frac{c_{3}(d s+s-d)\left(w^{2}\right)^{g}}{\left(1-s w^{2}\right)(1-s)} \frac{\left(s^{g}\right)^{2}}{N_{g}-1}+\frac{\left(1+s w^{2}\right)(d s+s-d)}{\left(1-s w^{2}\right)(s-1)} \frac{g s^{g}}{N_{g}-1} \\
& +\left[\frac{c_{3}\left(w^{2}\right)^{g}}{\left(w^{2}-1\right)\left(1-s w^{2}\right)}-\frac{s\left(1+s w^{2}\right)}{(1-s)^{2}\left(1-s w^{2}\right)}-\frac{c_{3} w^{2}}{\left(w^{2}-1\right)\left(1-s w^{2}\right)}-\frac{c_{3} s\left(w^{2}\right)^{g}}{(1-s)\left(1-s w^{2}\right)}\right. \\
& \left.+\frac{c_{3}(d+1)}{1-s w^{2}}+T_{t o t}(0)\right] \frac{s^{g}}{N_{g}-1}+\frac{s\left(1+s w^{2}\right)}{(1-s)^{2}\left(1-s w^{2}\right)} \frac{1}{N_{g}-1},
\end{aligned}
$$

where $c_{3}=d w\left(1+\frac{T_{t o t}(0)}{d}\right)-\frac{2 s w^{2}}{1-s w^{2}}$.

Recalling $s^{g}=\frac{(s-1) N_{g}}{d s+s-d}$, we have

$$
\begin{aligned}
\langle T\rangle_{g} & \approx \frac{c_{3}}{s w^{2}-1}\left(s w^{2}\right)^{g} \approx \frac{c_{3}}{s w^{2}-1}\left(\frac{s-1}{d s+s-d}\right)^{\log _{s}\left(s w^{2}\right)} N_{g}^{\log _{s}\left(s w^{2}\right)} \\
& \sim N_{g}^{\log _{s}\left(s w^{2}\right)} .
\end{aligned}
$$

Case (iii) : $w=1$ and $0<r<1$

The dominating term of $\langle T\rangle_{g}$ can be obtained as

$$
\begin{aligned}
\langle T\rangle_{g} & \approx \frac{c_{4}}{s-1}(s r)^{g} \approx \frac{c_{4}}{s-1}\left(\frac{s-1}{d s+s-d}\right)^{\log _{s}(s r)} N_{g}^{\log _{s}(s r)} \\
& \sim N_{g}^{\log _{s}(s r)}
\end{aligned}
$$

where $c_{4}=d r\left(1+\frac{T_{t o t}(0)}{d}\right)-\frac{2 s r}{1-s r}$.

Case $(i v): r=w=1$

The dominating term of $\langle T\rangle_{g}$ can be obtained as

$$
\begin{aligned}
\langle T\rangle_{g} & \approx \frac{c_{5}}{d s+s-d} \frac{\left(N_{g}\right)^{2}}{N_{g}-1} \approx \frac{c_{5}}{d s+s-d} N_{g} \\
& \sim N_{g},
\end{aligned}
$$

where $c_{5}=d\left(1+\frac{T_{t o t}(0)}{d}\right)-\frac{2 s}{1-s}$, and which is compared with that of Koch networks in Ref. [31].

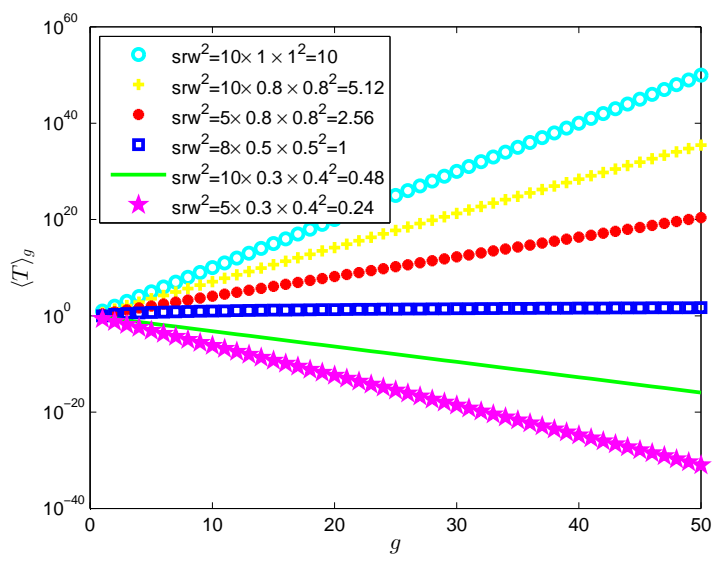

Figure 6: AWTT $\langle T\rangle_{g}$ versus $g$ on a semilogarithmic scale. 
When $s r w^{2}=1$, AWTT grows with increasing order $N_{g}$ as $\log N_{g}$ from Eq. (8). When $s r w^{2} \neq 1$, Eqs. (12-15) confirm that in large network, the leading behaviors of AWTT for the node- and edge- weighted fractal networks follow distinct scalings, with the trapping efficiency associated with the network size $N_{g}$, the number of copies $s$, the node-weight factor $w$ and the edge-weight factor $r$. Furthermore, in the large network, the AWTT grows as a power-law function of the network size $N_{g}$ with the exponent, represented by $\theta(s, r, w)=\log _{s}\left(s r w^{2}\right)$. From Eqs. (12-15), some results can be obtained as follows:

(i) When $0<s r w^{2}<1$, for a large system, i.e., $N_{g} \rightarrow \infty$, we can get $\langle T\rangle_{g} \rightarrow 0$. The result shows that the smaller $w$ is, the more quickly the walker arrives at the central node and the smaller $r$ is, the less the weighted time which the walker consumes is . That is to say, the trapping efficiency of the nodeand edge- weighted fractal networks is higher.

(ii) When $1<s r w^{2}<s$, for a large system, i.e., $N_{g} \rightarrow \infty$, we can get $\langle T\rangle_{g} \rightarrow \infty$. AWTT grows sublinearly with the network size $N_{g}$.

(iii) When $r=w=1\left(\right.$ i.e., $\left.s r w^{2}=s\right)$, for a large system, i.e., $N_{g} \rightarrow \infty$, we can get $\langle T\rangle_{g} \rightarrow \infty$. AWTT grows linearly with the network size $N_{g}$.

For the range of $g \leq 50$, average weighted trapping time $\langle T\rangle_{g}$ versus $g$ on a semilogarithmic scale is shown in Fig. 6. When $s r w^{2}=1$, AWTT grows with increasing order $N_{g}$ as $\log N_{g}$. When $s r w^{2} \neq 1$, the AWTT in large network grows as a power-law function of the network size $N_{g}$ with the exponent, represented by $\theta(r, w)=\log _{s}\left(s r w^{2}\right)$. If $r=w=1\left(i . e ., s r w^{2}=s\right)$, AWTT grows linearly with the system size $N_{g}$. If $1<s r w^{2}<s$, AWTT grows sublinearly with the system size $N_{g}$. If $s r w^{2}<1$, AWTT decreases gradually to 0 in the limit of large network size $N_{g}$. This also means that the efficiency of the trapping process depend on three main parameters: the number of copies $s>1$, node-weight factor $0<w \leq 1$ and edge-weight factor $0<r \leq 1$. Intuited by the highway networks, a theoretical model of node- and edge- weighted fractal networks is constructed. we have studied the trapping problem in a theoretical model. The result we have got shows that the smaller the value of $s r w^{2}$ is, the more efficient the trapping process is. However, the real traffic networks do not have the ideal fractal structure, that is to say, exact analytical results for the AWTT are obtained in the ideal mathematical model.

\section{Conclusions}

To explore the effect of the underlying structures, the number of copies, node weights and edge weights on the trapping efficiency, we have studied the trapping problem defined on the node- and edge- weighted fractal networks, concentrating on a particular case with the single trap positioned at the central node. Our results show that in large network, the leading behaviors of AWTT for the node- and edge- weighted fractal networks follow distinct scalings, with the trapping efficiency associated with the network size $N_{g}$, the number of copies $s$, the node-weight factor $w$ and the edge-weight factor $r$. Our analysis has indicated that AWTT grows with increasing order $N_{g}$ as $\log N_{g}$ when $s r w^{2}=1$. And in large network, the AWTT grows as a power-law function of the network size $N_{g}$ with the exponent, represented by $\theta(r, w)=\log _{s}\left(s r w^{2}\right)$ when $s r w^{2} \neq 1$. Specifically speaking, if $r=w=1\left(i . e ., s r w^{2}=s\right)$, AWTT grows linearly with the system size $N_{g}$; if $1<s r w^{2}<s$, AWTT grows sublinearly with the system size $N_{g}$; if $s r w^{2}<1$, AWTT decreases gradually to 0 in the limit of large network size $N_{g}$. This also means that the efficiency of the trapping process depend on three main parameters: the number of copies $s>1$, node-weight factor $0<w \leq 1$ and edge-weight factor $0<r \leq 1$. The smaller the value of $s r w^{2}$ is, the more efficient the trapping process is.

In the ideal mathematical model of node- and edge- weighted fractal networks, exact analytical results for the AWTT have been obtained. However, the real traffic networks do not have the ideal fractal structure. Real traffic networks are more random than fractal. It should be mentioned that we only studied the trapping problem in a theoretical model of node- and edge- weighted fractal networks, whether the conclusion also holds for random networks, which needs further investigations. 


\section{Acknowledgements}

The authors are grateful to the valuable comments from the anonymous reviewers, which are very useful to improve the paper quality.

Research is supported by the Humanistic and Social Science Foundation from Ministry of Education of China (Grants 14YJAZH012), National Natural Science Foundation of China (Nos. 11371329, 11471124), NSF of Zhejiang Province (No. LR13A010001) and Projects in Science and Technique of Ningbo Municipal (No. 2012B82003).

\section{References}

[1] Albert R, Barabási A L. Statistical mechanics of complex networks[J]. Rev. Modern. Phys, 2002,74(1); 47-54.

[2] Dorogovtsev S N and Mendes J F F. Evolution of networks: from biological nets to the internet and WWW[M]. Oxford: Oxford University Press, 2003.

[3] Achard S, Salvador R, Whitcher B, Suckling J and Bullmore E. A Resilient, Low-Frequency, SmallWorld Human Brain Functional Network with Highly Connected Association Cortical Hubs[J]. J. Neurosci, 2006, 26; 63-75.

[4] Zhou C, Zemanová L, Zamora G, Hilgetag C C and Kurths J. Hierarchical Organization Unveiled by Functional Connectivity in Complex Brain Networks [J]. Phys. Rev. Lett, 2006, 97(23); 238103-238115.

[5] Donges J F, Zou Y, Marwan N and Kurths J. The backbone of the climate network[J]. EPL, 2009, 87; 48007-48014.

[6] Donges J F, Schultz H C H, Marwan N, Zou Y and Kurths J. Investigating the topology of interacting networks[J]. Eur. Phys. J. B, 2011, 84; 635-651.

[7] Garlaschelli D and Loffredo M I. Structure and evolution of the world trade network[J]. Physica A, 2005,355(1); 138-144.

[8] Hidalgo C A, Klinger B, Barabási A L and Hausmann R. The Product Space Conditions the Development of Nations[J]. Science, 2007, 317; 482-487.

[9] Heitzig J, Donges J F, Zou Y, Marwan N, and Kurths J. Node-weighted measures for complex networks with spatially embedded, sampled, or differently sized nodes[J]. Eur. Phys. J. B, 2012, 85; 38-57.

[10] Zemp D C, Wiedermann M, Kurths J, Rammig A, and Donges J F. Node-weighted measures for complex networks with directed and weighted edges for studying continental moisture recycling[J]. EPL, 2014, 107; 58005-58010.

[11] Wiedermann M, Donges J F, Heitzig J, and Kurths J. Node-weighted interacting network measures improve the representation of real-world complex systems[J]. EPL, 2013, 102; 28007-28012.

[12] Zhang L L, Wang Y H, and Wang Q Y. Synchronization for time-varying complex dynamical networks with different-dimensional nodes and non-dissipative coupling[J]. Commun Nonlinear Sci Numer Simulat, 2015, 24; 64-74.

[13] Wu X J, Lu G T. Projective lag synchronization of the general complex dynamical networks with distinct nodes[J]. Commun Nonlinear Sci Numer Simulat, 2012, 17; 4417-4429.

[14] Lü L, Li C R, Chen L S, and Wei L L. Lag projective synchronization of a class of complex network constituted nodes with chaotic behavior[J].Commun Nonlinear Sci Numer Simulat, 2014, 19; 28432849.

[15] Solís-Perales G, Ruiz-Velázquez E, and Valle-Rodríguez D. Synchronization in complex networks with distinct chaotic nodes[J].Commun Nonlinear Sci Numer Simulat, 2009, 14; 2528-2535.

[16] Barrat A, Barthélemy M, Pastor-Satorras R, and Vespignani A. The architecture of complex weighted networks[J]. Proc Natl Acad Sci USA, 2004, 101; 3747-3752.

[17] Cai G L, Yao Q, and Shao H J. Global synchronization of weighted cellular neural network with timevarying coupling delays[J]. Commun Nonlinear Sci Numer Simulat, 2012, 17; 3843-3847. 
[18] Krause A E, Frank K A, Mason D M, Ulanowicz R E and Taylor W W. Compartments revealed in food-web structure[J]. Nature, 2003, 426; 282-285.

[19] Pastor-Satorras R and Vespignani A. Evolution and Structure of the Internet-A Statistical Physics Approach[M]. Cambridge: Cambridge University Press, 2004.

[20] Carletti T, Righi S. Weighted fractal networks[J]. Physica A, 2010, 389; 2134-2142.

[21] Dai M F, Ye D D, Hou J, and Li X Y. Scaling of average weighted receiving time on double-weighted Koch networks[J]. Fractals, 2015, 23(02); 1550011-1550017.

[22] Barnsley M. Fractals Everywhere[M]. Academic Press, London, 1988.

[23] Edgar G A. Measure, Topology and Fractal Geometry[M]. In: UTM, Springer-Verlag, New York, 1990.

[24] Singh S L, Mishra S N, and Sinkala W. A new iterative approach to fractal models[J]. Commun Nonlinear Sci Numer Simulat, 2012, 17; 521-529.

[25] Singh S L, Mishra S N, and Sarika J. Orbit of an image under iterated system[J]. Commun Nonlinear Sci Numer Simulat, 2011, 16; 1469-1482.

[26] Wei D J, Liu Q, Zhang H X, Hu Y, Deng Y and Mahadevan S K. Box-covering algorithm for fractal dimension of weighted networks[J]. Scientific Reports, 2013, 03; 3049-3055.

[27] Huang Y, Gu Y, and Wang S. How to get the reliable fractal dimension by the box counting[J]. Communications in Nonlinear Science and Numerical Simulation, 1996, 02; 22-27.

[28] Chaoming Song, Lazaros K Galios, Shlomo Havlin, and Hernán A Makse. How to calculate the fractal dimension of a complex network: the box covering algorithm[J]. Journal of Statistical Mechanics: Theory and Experiment, 2007, 03; 03006-03021.

[29] Christian M S, Tobias A K, Josá S A J, and Hans J H. Box-covering algorithm for fractal dimension of complex networks[J]. Physical Review E, 2012 86(1); 016707-016717.

[30] Volchenkov D. Random walks and flights over connected graphs and complex networks[J]. Commun Nonlinear Sci Numer Simulat, 2011, 16; 21-55.

[31] Zhang Z Z, Zhou S G, Xie W L, Chen L C, Lin Y and Guan J H. Standard random walks and trapping on the Koch network with scale-free behavior and small-world effect[J]. Phys. Rev. E, 2009, 79; 061113061124 . 